\title{
El valor social de la educación: a modo de introducción
}

\author{
Jesús Miguel Jornet Meliá ${ }^{1}$ \\ Carlos Sancho-Álvarez ${ }^{2}$
}

La crisis económica sufrida en los últimos años, y en concreto en España, ha transmitido una cierta desconfianza en la sociedad acerca del valor que posee la educación para la integración y promoción socio-laboral de los jóvenes, así como en relación al impacto que ésta tiene para prevenir de la exclusión social a lo largo de la vida.

Por desgracia en España, aún en la actualidad, que se considera que la crisis económica está en proceso de superación, hay una gran cantidad de jóvenes que se les identifica como "sobre-formados" y que no encuentran un empleo adecuado a su formación. Unos acaban trabajando en empleos que requieren menor formación académica, mientras que otros acaban en el paro o teniendo que emigrar a otros países. Personalmente es un problema gravísimo, pero políticamente también lo es, pues resulta que la inversión educativa se convierte de este modo en un gasto, tanto personal como económico. Adicionalmente, la creencia de que la educación conlleva una mayor seguridad para el bienestar social está en gran medida también en crisis.

No obstante, la Educación se ha considerado tradicionalmente como un factor que aporta mejoras para el desarrollo personal y la transformación social. Sistemáticamente se observa a partir de diversos indicadores sociales que las personas que tienen mejores niveles educativos también presentan mayores niveles socio-económicos y menor probabilidad de exclusión social a lo largo de la vida. Estos indicadores presentan la misma tendencia en el Informe Panorama de la Educación (Education at a Glance) que periódicamente realiza la OCDE. Asimismo, las diferencias que se dan entre colectivos más y menos favorecidos se dan de forma concomitante entre los niveles socioeconómicos y educativos.

Por último, señalar que los países que destinan mayores recursos a educación, sea desde el ámbito público y/o el privado, también son aquéllos que presentan mayor nivel de desarrollo. Esta asociación, no es casual. De hecho, desde los primeros estudios (como, por ejemplo, el de Coleman et al., 1966) en los que se intentaban identificar los factores que explicaran mejor $-\mathrm{o}$ incluso predijeran- los logros o resultados académicos, el nivel socio-económico y cultural de las familias se ha mostrado como el más potente, por encima de factores escolares. Este tipo de resultados se sigue observando a partir de investigaciones realizadas con datos recabados en evaluaciones nacionales e internacionales a gran escala. En otros estudios, con enfoques metodológicos más micro-analíticos, no obstante, se puede identificar mejor el valor diferencial que tienen otros factores personales, familiares y escolares. Así, ello no quiere decir que la escuela sea un instrumento que no compense ni mejore las condiciones de personas y colectivos vulnerables, pero pone de manifiesto que la situación social, representada en lo económico y en lo cultural de las familias y la sociedad en general, es un factor clave que acompaña al trabajo escolar para la obtención de los logros educativos condicionándolos, incluso, positiva o negativamente.

Por todo ello, cuando se aborda el análisis de la calidad educativa, sea a nivel personal, de centros o de sistemas, normalmente se recaba información de indicadores que, de manera directa o indirecta, puedan poner de manifiesto el valor que se le reconoce u otorga a la educación. El contexto social indudablemente es un facilitador o un obstaculizador del rol que se le dé a la educación y éste, a su vez, de los posibles logros o resultados. De ahí que, en muchos estudios sobre políticas educativas, se informe sobre el porcentaje del Producto Interior Bruto (PIB) o del PIB per cápita, que

\footnotetext{
${ }^{1}$ Dr. en Educación, Catedrático de Medición y Evaluación Educativas, Dpto. MIDE de la Universitat de València (España). Grupo de Investigación GemEduCo y de Innovación InnovaMIDE (www.uv.es/gem). Contacto: jornet@uv.es.

2 Dr. en Educación, acreditado Profesor Contratado Doctor, Universidad Antonio de Nebrija (España). Grupo de Investigación GemEduCo y de Innovación InnovaMIDE (www.uv.es/gem). Contacto: csancho@,nebrija.es.
} 
destinan los gobiernos a la Educación y de otros indicadores económicos como demostración de la importancia que se le da, desde la acción de gobierno, a la educación entendida como elemento de transformación y bienestar personal y social.

Desde otra perspectiva de análisis, en estudios sobre evaluación de la calidad de sistemas educativos se observa que es frecuente recoger información acerca de las expectativas y aspectos motivacionales que los escolares o sus familias tienen hacia la educación; por ejemplo, una pregunta habitual dirigida a familias como "¿hasta qué nivel desearian que estudien sus hijos?" presenta, normalmente, tendencias de asociación positiva con los resultados escolares.

Cuando comenzamos a desarrollar el modelo de evaluación de instituciones y sistemas educativos, basado en el concepto de Cohesión Social (Jornet, 2010; 2012), este tipo de apreciaciones nos llevaron a considerar el Valor Social de la Educación (VSE) como un indicador complejo, es decir, un constructo teórico que podría ser medido a nivel personal, social y político.

En el trabajo de Jornet, Perales y Sánchez-Delgado (2011) planteamos que el valor social que se otorga a la educación podía abordarse, a nivel evaluativo, desde dos perspectivas: el valor que le otorgan las políticas públicas a la educación, y el valor que le reconocen las personas (alumnado, familias y profesorado). En el primer caso, nos referimos al Valor Social Objetivo de la Educación (VSE-O). Por su parte, en el segundo caso nos referimos al Valor Social Subjetivo (VSE-S) de la Educativo.

Esta doble consideración, nos permite reflexionar acerca de qué indicios e indicadores pueden identificarse para evaluar el valor social de la educación en ambos planos de análisis. En los dos casos, la reflexión nos orientó hacia un planteamiento metodológico de forma que permitiera abordar la importancia del valor social de la educación desde un planteamiento holista, globalizador; es decir, como un constructo complejo, producto de indicadores simples como, por ejemplo, un grupo de preguntas como la mencionada anteriormente dirigidas a escolares o a familias. De igual forma, para el análisis de políticas educativas, sería posible teorizar acerca de las dimensiones del valor social que se da a la educación desde un enfoque político analizando indicadores simples y cómo se relacionan entre sí configurando un constructo teórico global.

En este número monográfico se invitó a la comunidad científica a presentar sus aportaciones acerca del valor social que se otorga a la educación, desde la posición teórico-metodológica que cada cual tuviera al respecto. A partir de la convocatoria realizada por la revista se han recibido diversas contribuciones. De ellas, se presentan siete de gran interés como muestra del estado de la cuestión y de los diversos enfoques que se pueden dar en el tratamiento de esta temática.

Las tres primeras aportaciones se refieren a la calidad, la evaluación y la política educativas, como indicadores del VSE-O que se ha dado a la educación en las últimas décadas. Creemos que es importante destacar que precisamente esta preocupación ha cobrado una especial relevancia en América Latina. Por ello, no es de extrañar que los tres primeros trabajos provengan de autores latinoamericanos.

En el primero de ellos, presentado por el Dr. Felipe Martínez Rizo, se analiza la creciente preocupación por la calidad educativa que se ha observado en muchos países y que se ha manifestado especialmente a partir del incremento de evaluaciones a gran escala, orientadas a informar la política educativa. El autor presenta sus posiciones acerca de la necesidad de abordar las evaluaciones de la calidad superando planteamientos simplistas y falaces que se han extendido en esta área. El segundo trabajo del monográfico es el que presenta la Dra. Ivana G. Zacarias, en el que analiza de forma exhaustiva la evolución de los enfoques, instituciones y proyectos evaluativos acerca de la calidad educativa que se han ido desarrollando en Latinoamérica y, el tercero, aportación realizada por el Dr. Pablo Daniel García, sobre la provisión de recursos como estrategia para la mejora de los logros escolares en las políticas educativas en América Latina. Estos trabajos ponen de manifiesto el interés social que se ha ido instaurando sobre el rol de la educación como motor de desarrollo social y "ascensor social" y, desde el punto de vista científico-académico, el hecho de que el análisis del impacto y relevancia de la educación en la sociedad es un fenómeno que debe ser analizado desde la confluencia de diversas especialidades pedagógicas, como la Política Educativa, la Educación Comparada, la Medición, Evaluación e Investigación Educativas, así como desde la Sociología y la Economía. 
El segundo grupo de trabajos que aquí se presentan son una pequeña muestra de las diferentes facetas que pueden considerarse al investigar sobre el valor social subjetivo que se da a la educación. Tal como mencionamos anteriormente, el VSE-S en definitiva es una percepción que se construye a partir de experiencias que se dan en el ámbito personal, escolar, familiar y social. La persona acaba valorando la educación en función de diferentes vivencias, lo que constituye que sea un constructo que pueda y deba ser analizado en relación con otros constructos personales, escolares y sociales. La metodología, por tanto, es necesariamente diversa, abarcando desde estudios fundamentalmente de corte cuantitativo hasta otros cualitativos, sin olvidar los de complementariedad metodológica.

El primero de los trabajos que se presentan en este segundo grupo es la aportación de las Dras. $\mathrm{M}^{\mathrm{a}}$ Jesús Perales, Mercedes Bisquert y Margarita Bakieva. En estudios anteriores, las autoras trabajaron sobre estilos educativos familiares y el modo de evaluarlos en familias de escolares de educación primaria y secundaria obligatoria españoles, desarrollando y validando escalas a tal efecto. En este caso, nos presentan un análisis cuantitativo acerca de las relaciones que puedan darse entre el VSE-S y los estilos educativos familiares. Las evidencias aún son escasas, pero es muy interesante acercarse a un estudio entre constructos que, muy probablemente, deben estar relacionados y podría ser avalado por resultados que son difíciles de recabar, tanto por el modo en que puedan ser medidos los estilos educativos familiares como el VSE-S asociado a ellos.

El segundo de los trabajos que conforman este bloque relativo al VSE-S, es el de los Dres. F. Javier Murillo y Nina Hidalgo. Se trata de un estudio fenomenográfico sobre cómo se construye a partir de la enseñanza la percepción de la justicia social. Cuando analizamos el VSE-S la dimensión de percepción de justicia social encontramos que está íntimamente relacionada con él, a la par que está asociada a las vivencias que se dan en la escuela, así como al trabajo que desde ella se realiza para que el alumnado pueda desarrollar criterios de valoración acerca de la justicia social.

El siguiente trabajo, presentado por los Dres. Brenda I. Boroel, José Sánchez-Santamaría, Karla D. Morales y Patricio S. Henríquez nos ofrecen los resultados de la evaluación de un programa de acompañamiento escolar basado en un proceso de tutoría. Su finalidad es analizar la capacidad de este tipo de programas orientados desde el concepto de equidad educativa. El último trabajo que compone el monográfico ha sido realizado por las Dras. Judith Quintano, Sonia Ortega y María Tejedor. Nos ofrecen el estudio de las aportaciones de programas universitarios dirigidos a personas mayores. La educación a lo largo de la vida no tiene únicamente un valor social vinculado a la empleabilidad, sino que constituye un instrumento de apoyo personal, cultural, y social en diversos momentos de la transición por las diversas etapas vitales. Un estudio de caso pone de manifiesto el VSE-S que implica este tipo de programas, cada vez más frecuentes en las universidades.

Para finalizar, agradecer a la Revista Fuentes la oportunidad que ha brindado a la comunidad científico-académica para abordar esta temática y a los autores que han colaborado con sus trabajos en este número.

Referencias Bibliográficas.

Coleman, J. S., Campbell, E. Q., Hobson, C. J., McPartland, J., Mood, A. M., Weinfeld, F. D., y York, R. L. (1966). Equality of Educational Opportunity. Washington: Office of Education.

Jornet, J.M. (2010). Dimensiones docentes y cohesión social: reflexiones desde la evaluación. Ponencia presentada en el II Coloquio de la Red Iberoamericana de Investigación sobre la Docencia (RIIED), Valencia, Septiembre. Revista Iberoamericana de Evaluación Educativa (2012)- Vol. 5(1), 349-362. Accesible en: http://www.rinace.net/riee/numeros/vol5-num1_e/art27.pdf

Jornet, J.M., Perales, M.J. y Sánchez-Delgado, P. (2011). El Valor Social de la educación: entre la subjetividad y la Objetividad. Consideraciones teórico-metodológicas para su evaluación. Revista Iberoamericana de Evaluación Educativa, 4(1), 51-77. Accesible en: http://www.rinace.net/riee/numeros/vol4-num1/art3.pdf 